UCR L-8713 (Rev.)

\author{
UNIVERSITY OF CALIFORNIA \\ Lawrence Radiation Laboratory \\ Berkeley, Calif or nia \\ Contract No. W-7405-eng-48
}

\title{
FREE ENERGY FUNCTIONS FOR GASEOUS MONOXIDES
}

L. Brewer and M.S. Chandrasekharaiah

June 1960

This document is

PUBLICLY RELEASABLE

B Staelo

Authorizing Official

Date:

$6 / 16 / 06$ 


\section{DISCLAIMER}

This report was prepared as an account of work sponsored by an agency of the United States Government. Neither the United States Government nor any agency Thereof, nor any of their employees, makes any warranty, express or implied, or assumes any legal liability or responsibility for the accuracy, completeness, or usefulness of any information, apparatus, product, or process disclosed, or represents that its use would not infringe privately owned rights. Reference herein to any specific commercial product, process, or service by trade name, trademark, manufacturer, or otherwise does not necessarily constitute or imply its endorsement, recommendation, or favoring by the United States Government or any agency thereof. The views and opinions of authors expressed herein do not necessarily state or reflect those of the United States Government or any agency thereof. 


\section{DISCLAIMER}

Portions of this document may be illegible in electronic image products. Images are produced from the best available original document. 
Printed in USA. Price 50 cents. Available from the office of Technical Services

U. S. Department of Commerce Washingt on 25 , D. C. 


\title{
FREE ENERGY FUNCTIONS FOR GASEOUS MONOXIDES
}

L. Brewer and M. S. Chandrasekharaiah

\section{Lawrence Radiation Laboratory and Department of Chemistry \\ University of California, Berkeley 4, California}

\begin{abstract}
Free energy functions for gaseous monoxides have been calculated from presently available spectroscopic results. However, the electronic contributions to the free energy functions have been estimated. A simple ionic model has been assumed since the molecular electronic states for most of these oxides are not known. In some instances where experimental data are insufficient to calculate the interatomic distances and the equilibrium frequencies of vibrations, they have been estimated. The results of these calculations are tabulated for $500^{\circ}$ intervals from room temperature up to $3000^{\circ} \mathrm{K}$.
\end{abstract}




\section{FREE ENERGY FUNCTIONS FOR GASEOUS MONOXIDES}

\section{Brewer and M. S. Chandrasekharaiah \\ Lawrence Radiation Laboratory and Department of Chemistry University of California, Berkeley 4, California}

June 1960

In predicting the stability of refractory oxides at high temperatures, thermodynamic data for both the condensed and gaseous phases at these temperatures will be of great help. An ideal situation will be tabulations of accurate thermodynamic functions for all oxides. Unfortunately, presently available experimental data are insufficient to do a thorough job of calculating these thermodynamic functions. Hence, one has to estimate them in many cases. For such estimates, different investigators have used different approximations and it is not always quite clear what approximations have been made in their calculations. Also, the combination of thermodynamic functions calculated by different methods introduces unnecessary errors.

Even though the presently available experimental data are insufficient for accurate calculations of thermodynamic quantities for all oxides, tabulation of selfconsistent, though approximate, thermodynamic functions for the oxides would eliminate many of these difficulties.

In recent years, such compilations of thermodynamic data for elements, ${ }^{1}$ for condensed oxides ${ }^{2,3}$ etc., have been published. Brewer ${ }^{4}$ has calculated the dissociation energies for gaseous metal monoxides. Because of the importance of gaseous monoxides in the vaporization of metal oxides and as a continuation of similar programs in this laboratory, free energy functions of the gaseous monoxides are calculated from the available spectroscopic data. Results are tabulated for $500^{\circ}$ intervals from room temperature up to $3000^{\circ} \mathrm{K}$.

Emission spectra for many of these diatomic molecules have been analyzed and the molecular constants have been evaluated. The excellent compilation of Rosen ${ }^{5}$ et al, covers the literature survey up to 1951. Recently, Sutton ${ }^{6}$ et al, published a comprehensive report on the internuclear distances. Liberal use of these tabulations of the molecular constants have been made in these calculations. In spite of voluminous reported results in the literature for diatomic molecular spectra, estimates of $r_{e}$ and $\omega_{e}$ values had to be made for a number of oxides. The method of estimation is fairly simple and straightforward. 
A. Calculation of Electronic Contributions

Experimental results to fix the molecular electronic states of these oxides are lacking, particularly so in case of transition metal oxides. In many instances even the ground state is not firmly established. Hence the electronic contribution to the partition function has to be estimated in almost all cases. A simple ionic model approximation has been assumed in calculating the free energy functions.

To make the calculation uniform, only estimated values for the electronic contributions to the free energy functions are employed for all oxides. It is assumed that the molecular states may be approximated by the energy levels of the corresponding +2 metal ions and that the approach of an oxide ion causes no drastic perturbations. The electronic partition functions are thus approximated by the electronic partition functions of the free gaseous ions. Electronic partition functions are calculated from the energy levels of the corresponding cations (+2) taken from Moore $\mathrm{s}$ tabulation. 7 Table 1 contains the results of these calculations.

B. Calculation of Rotational and Vibrational Contributions

In calculating rotational and vibrational partition functions, $r_{e}$ and $\omega_{e}$ values are taken from References (5) and (6) whenever available. But for a number of oxides, rotational analysis of spectra are incomplete and hence $r_{e}$ values have to be estimated. Where the $\omega_{e}$ values for the molecules are available, interatomic distances are approximated by Badger's rule $e^{8}$ from the force constant data. In a few cases, both $r_{e}$ and $\omega_{e}$ values are estimated by interpolating between the neighboring molecules in the periodic table of elements. The $r_{e}$ and $\omega_{e}$ values employed in this calculation are given in Table 2 . The ground state values of $r_{e}$ and $\omega_{e}$ were also used for the excited electronic states.

So far, there have been no experimental results for any of the alkali oxides. All molecular constants are estimated. In evaluating electronic contributions to free energy functions, a modification in the method of estimating is made. Instead of approximating the molecular levels with those of the +2 cations, it is approximated with the energy levels of $0^{\infty}$ ion or its isoelectronic fluorine atom. Thus both the alkali metal and oxygen are considered to be in oxidation states of +1 and -1 , respectively.

Tables 3 and 4 contain the rotational and vibrational contribution to the free energy function of oxides respectively. The calculation of translation contribution is simple and straightforward. Table 5 contains these results. The total free energy functions for the gaseous monoxides are given in Table 6 . 


\section{REFERENCES}

1. D. R. Stull and G. C. Sinke, "Thermodynamic Properties of the Elements," No. 18 of the Advances in Chemistry Series. American Chemical Society, 1956.

2. J. L. Margrave, T. J. Katz, R. Polansky, M. S. Chandrasekharaiah, R. G. EhI, S. P. Randall and R. L. Tallman, Unpublished Tables, University of Wisconsin (1953-1956).

3. J. P. Coughlin, "Heats and free energies of formation of inorganic oxides," Bureau of Mines Bulletin No. 542, Washington, 1954.

4. Leo Brewer, "Dissociation energies of oxides," University of California Lawrence Radiation Laboratory Report, UCRL-8356 (1958).

5. B. Rosen, "Constantes selectionnées données spectroscopiques concernant les molécules diatomiques." Hermann and Cie, Paris, 1951.

6. L. E. Sutton, "Tables of interatomic distances and configuration in molecules and ions," The Chemical Society, London, 1958.

7. C. E. Moore, "Atomic Energy Levels," National Bureau of Standards Bulletin No. 467, Vols. I, II, and III, Washington, 1949-1958.

8. R. M. Badger, J. Chem. Phys. 3, 710, (1935).

9. A. Lagerquist and Lars-Erik Selin, Naturwissenschaften 42, 65 (1955).

10. Masatoshi Ninomiya, J. Phys. Soc. Japan 10, 829 (1955).

11. K. Suryanarayana Rao, Nature 173, 1240 (1954); Proc. Nat. Inst. Sc1. India 21A, 188 (1955).

12. S. G. Krishna Murthy, Proc. Phys. Soc. (London) 64A, 852 (1951).

13. D. Premaswarup, Current Science 24, 45 (1955); Ind. J. Phys. 29, 109 (1955).

14. A. Gatterer and S. G. Krishnamurthy, Nature 169, 543 (1952). 
Table 1

$$
-\left(\frac{\mathrm{F}_{\mathrm{T}}{ }^{\mathrm{O}}-\mathrm{H}_{\mathrm{O}}{ }^{\mathrm{O}}}{\mathrm{T}}\right)_{\mathrm{ele}} \text { for oxides in e.u. }
$$

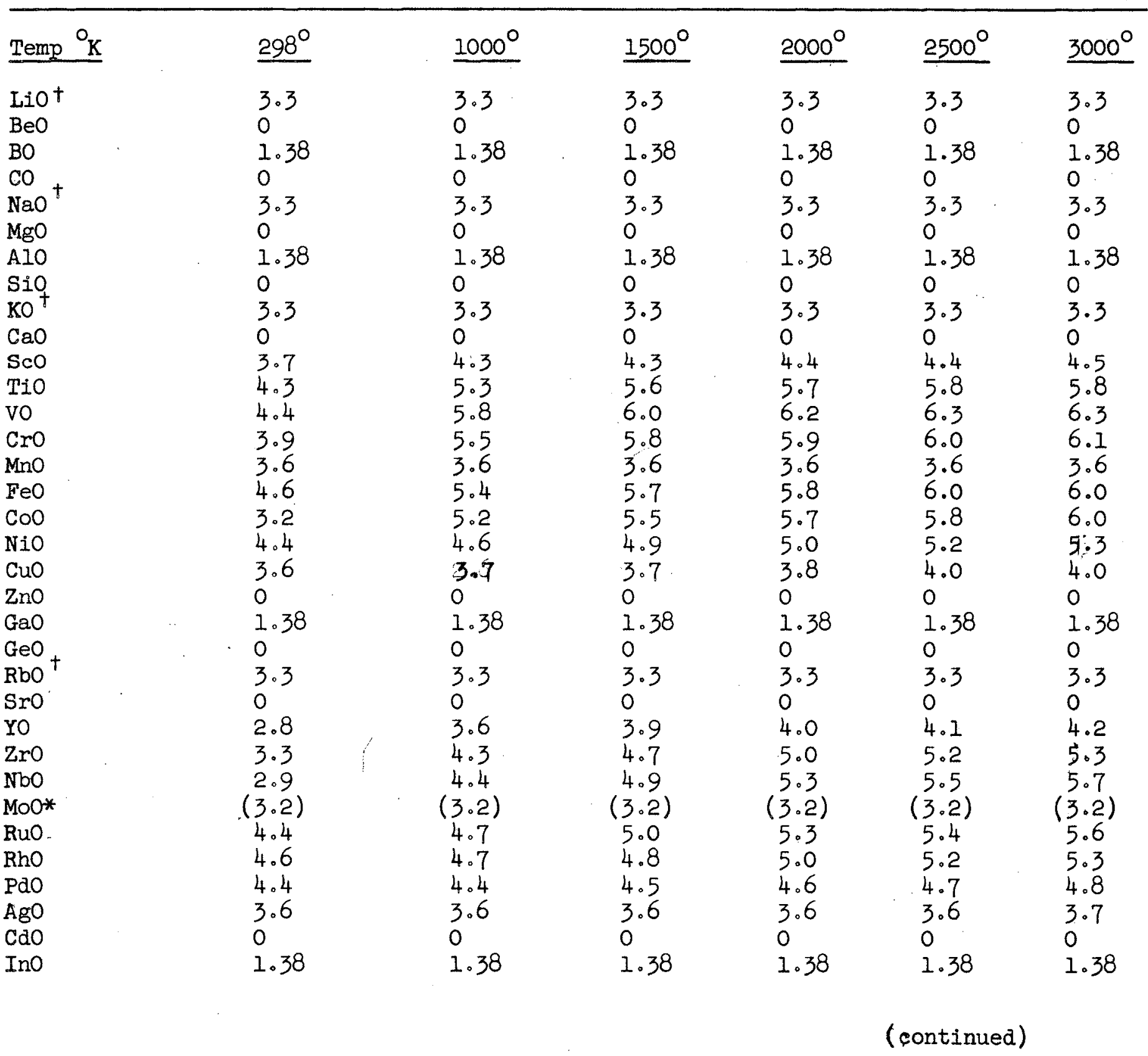


Table 1

(continued)

$$
-\left(\frac{F_{T}^{\circ}-H_{0}^{\circ}}{T}\right)_{e l e} \text { for oxides in e.u. }
$$

\begin{tabular}{|c|c|c|c|c|c|c|}
\hline Temp ${ }^{O_{K}}$ & $298^{\circ}$ & $1000^{\circ}$ & $1500^{\circ}$ & $2000^{\circ}$ & $2500^{\circ}$ & $3000^{\circ}$ \\
\hline $\mathrm{SnO}$ & 0 & 0 & 0 & 0 & 0 & 0 \\
\hline $\mathrm{CsO}^{+}$ & 3.3 & 3.3 & 3.3 & 3.3 & 3.3 & 3.3 \\
\hline $\mathrm{BaO}$ & 0 & 0 & 0 & 0 & 0 & 0 \\
\hline $\mathrm{LaO}$ & 2.8 & 3.0 & 3.3 & 3.5 & 3.7 & 3.8 \\
\hline $\mathrm{HfO}$ & 3.1 & 3.1 & 3.1 & 3.1 & 3.1 & 3.1 \\
\hline $\mathrm{TaO}^{*}$ & $(4.1)$ & $(4.1)$ & $(4.1)$ & $(4.1)$ & $(4.1)$ & $(4.1)$ \\
\hline WO* & $(3.2)$ & $(3.2)$ & $(3.2)$ & $(3.2)$ & $(3.2)$ & $(3.2)$ \\
\hline $\operatorname{ReO} *$ & $(3.6)$ & $(3.6)$ & $(3.6)$ & $(3.6)$ & $(3.6)$ & $(3.6)$ \\
\hline OsO & 4.4 & 4.4 & 4.4 & 4.4 & 4.4 & 4.4 \\
\hline IrO & 4.4 & 4.4 & 4.4 & 4.4 & 4.4 & 4.4 \\
\hline Pto & 4.4 & 4.4 & 4.4 & 4.4 & 4.4 & 4.4 \\
\hline AuO & 3.6 & 3.6 & 3.6 & 3.6 & 3.6 & 3.6 \\
\hline $\mathrm{HgO}$ & 0 & 0 & 0 & 0 & 0 & 0 \\
\hline T10 & $1: 38$ & 1.38 & 1.38 & 1.38 & 1.38 & 1.38 \\
\hline $\mathrm{PbO}$ & 0 & 0 & 0 & 0 & 0 & 0 \\
\hline $\mathrm{B} 10$ & 1.38 & 1.38 & 1.38 & 1.38 & 1.38 & 1.38 \\
\hline
\end{tabular}

*Estimated by comparing the other elements of the same group. No analysis of electronic levels for $\mathrm{M}^{+2}$ ions are given。

${ }^{\dagger}$ Estimated assuming $\mathrm{M}^{+} \mathrm{O}^{-}$and using $\mathrm{F}$ levels for $\mathrm{O}^{-}$as described in text. 
Table 2

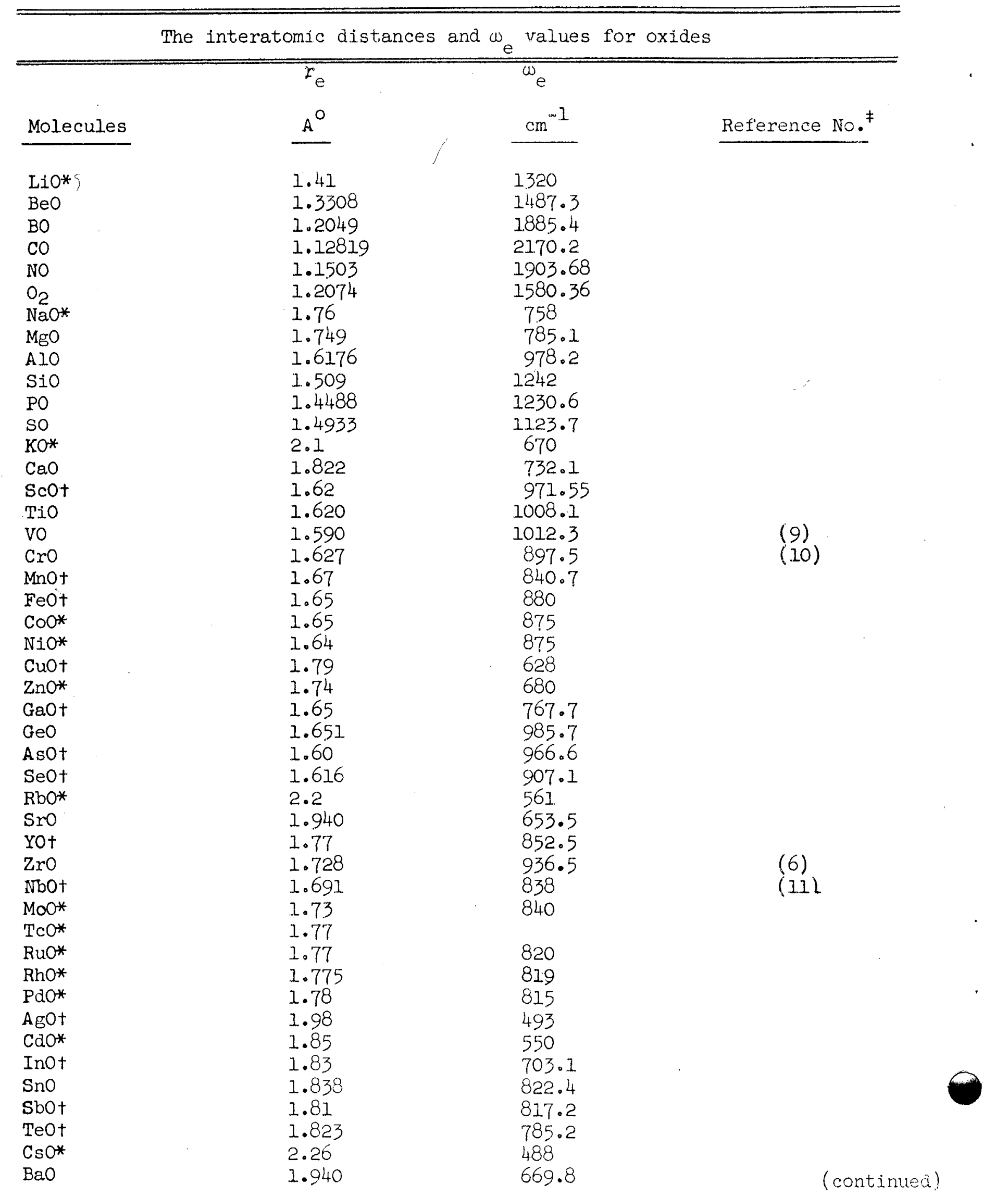


Table 2

(continued)

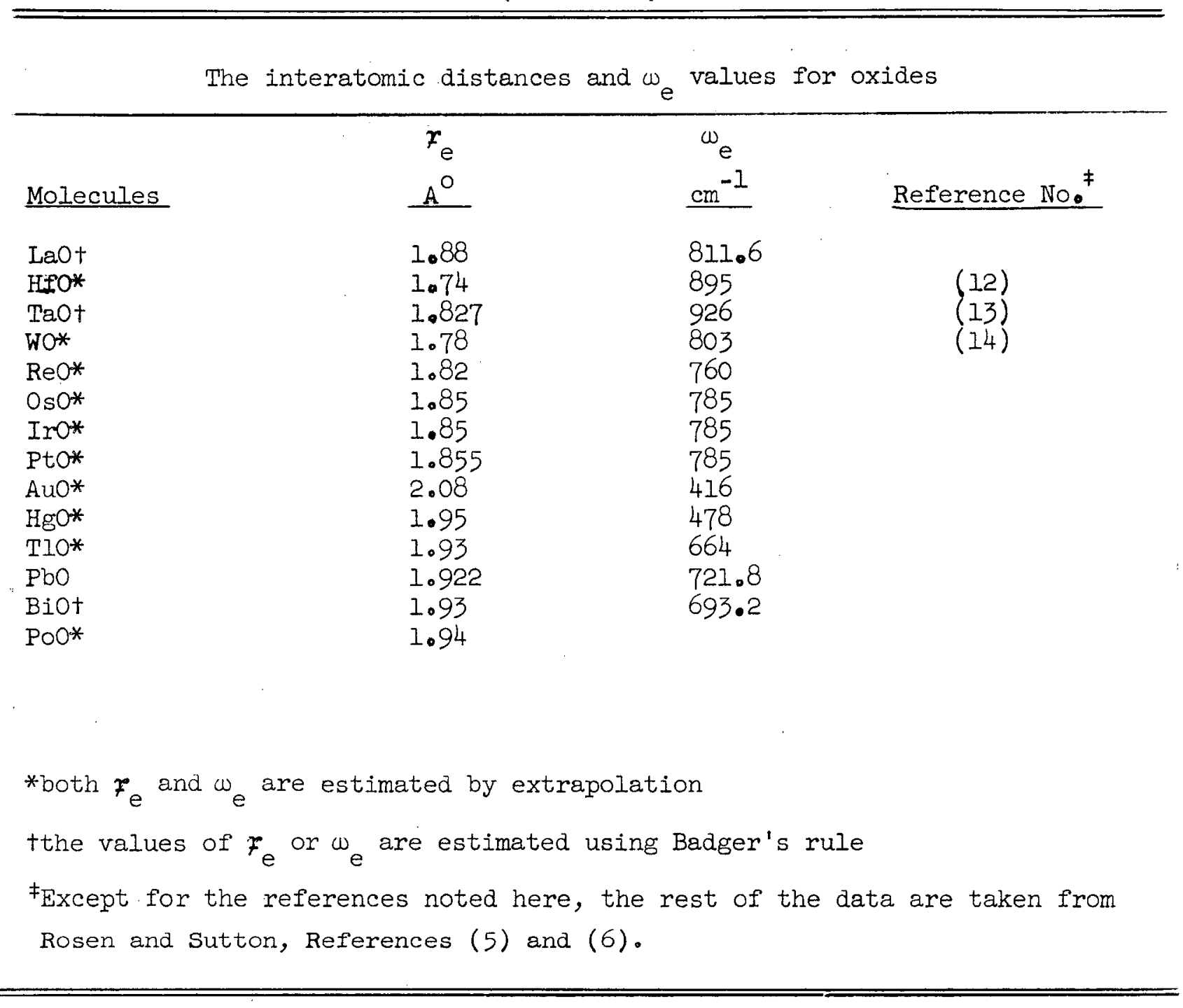


Table 3

$$
-\left(\frac{F_{T^{0}}-H_{0}^{0}}{T}\right)_{\text {rot }} \text { for oxides }
$$

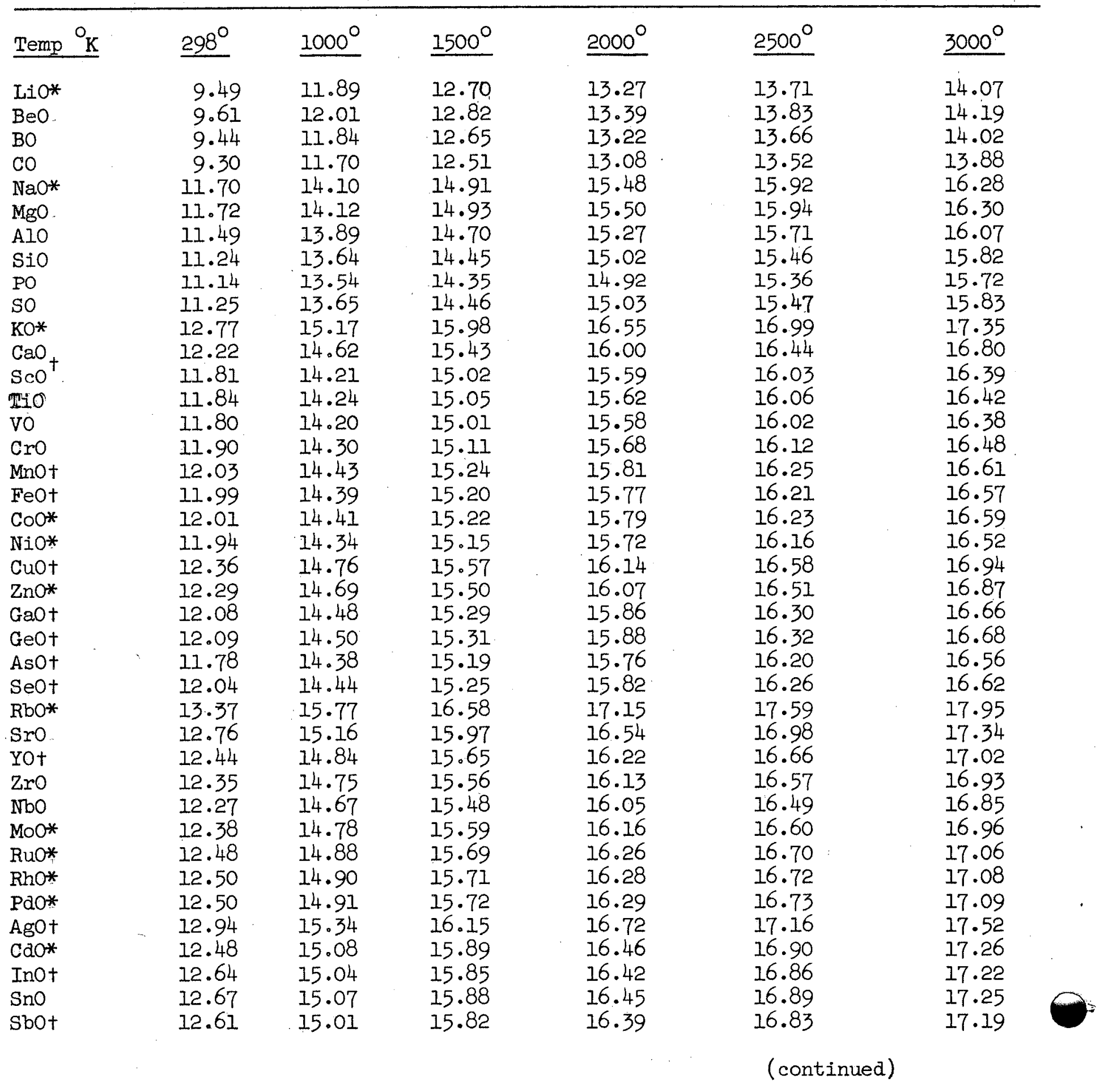


Table 3

(continued)

$$
-\left(\frac{\mathrm{F}_{\mathrm{T}}{ }^{\circ}-\mathrm{H}_{\mathrm{O}}^{\circ}}{\mathrm{T}}\right)_{\text {rot }} \text { for oxides }
$$

\begin{tabular}{|c|c|c|c|c|c|c|}
\hline Temp ${ }^{\circ} \mathrm{K}$ & $298^{\circ}$ & $1000^{\circ}$ & $1500^{\circ}$ & $2000^{\circ}$ & $2500^{\circ}$ & $3000^{\circ}$ \\
\hline TeOt & 12.65 & 15.05 & 15.86 & 16.43 & 16.87 & 17.24 \\
\hline $\mathrm{CsO}_{5} *$ & 13.52 & 15.92 & 16.73 & $17 \cdot 30$ & 17.74 & 18.10 \\
\hline $\mathrm{BaO}$ & 12.91 & 15.32 & 16.12 & 16.69 & 17.14 & 17.50 \\
\hline $\mathrm{LaOt}$ & 12.79 & 15.19 & 16.00 & 16.57 & 17.01 & 17.37 \\
\hline $\mathrm{HfO} *$ & 12.53 & 14.93 & 15.74 & 16.31 & 16.75 & 17.12 \\
\hline $\mathrm{TaO}^{*}$ & 12.73 & 15.13 & 15.94 & 16.51 & 16.95 & 17.31 \\
\hline WO* & 12.62 & 15.03 & 15.83 & 16.41 & 16.85 & 17.21 \\
\hline $\operatorname{ReO*}$ & 12.71 & 15.12 & 15.92 & 16.50 & 16.94 & $17 \cdot 30$ \\
\hline $\mathrm{OsO}^{*}$ & 12.78 & 15.18 & 16.00 & 16.56 & 17.00 & 17.37 \\
\hline $\operatorname{IrO} *$ & 12.78 & 15.19 & 16.00 & 16.57 & 17.00 & 17.37 \\
\hline PtO* & 12.80 & 15.20 & 16.01 & 16.58 & 17.02 & 17.38 \\
\hline AuO* & 13.27 & 15.67 & 16.48 & 17.05 & 17.49 & 17.85 \\
\hline $\mathrm{HgO} *$ & 13.06 & 15.46 & 16.27 & 16.84 & 17.28 & 17.64 \\
\hline T10* & 12.96 & 15.36 & 16.17 & 16.74 & 17.18 & 17.55 \\
\hline $\mathrm{PbO}$. & 12.94 & 15.35 & 16.15 & 16.73 & 17.17 & 17.53 \\
\hline $\mathrm{BiO}$ & 12.96 & 15.37 & 16.17 & 16.74 & 17.19 & 17.55 \\
\hline PoO* & 12.99 & 15.39 & 16.20 & 16.77 & 17.21 & $17 \cdot 57$ \\
\hline $\mathrm{RaO}^{*}$ & 13.08 & 15.48 & 16.29 & 16.86 & $17 \cdot 30$ & 17.67 \\
\hline $\mathrm{AcO}^{*}$ & 12.96 & 15.36 & 16.17 & 16.74 & 17.18 & 17.54 \\
\hline Tho & 12.96 & 15.36 & 16.17 & 16.74 & 17.18 & 17.54 \\
\hline UO* & 12.96 & 15.36 & 16.16 & 16.73 & 17.17 & 17.53 \\
\hline
\end{tabular}

${ }^{*} r_{e}$ are estimated by comparing with the neighbors.

$+r_{e}$ obtained from Badger rule approximation of force constant. 
Table 4

$$
-\left(\frac{\mathrm{F}_{\mathrm{T}}^{\mathrm{O}}-\mathrm{H}_{\mathrm{O}}^{\mathrm{O}}}{\mathrm{T}}\right)_{\mathrm{vib}} \text { for oxides }
$$

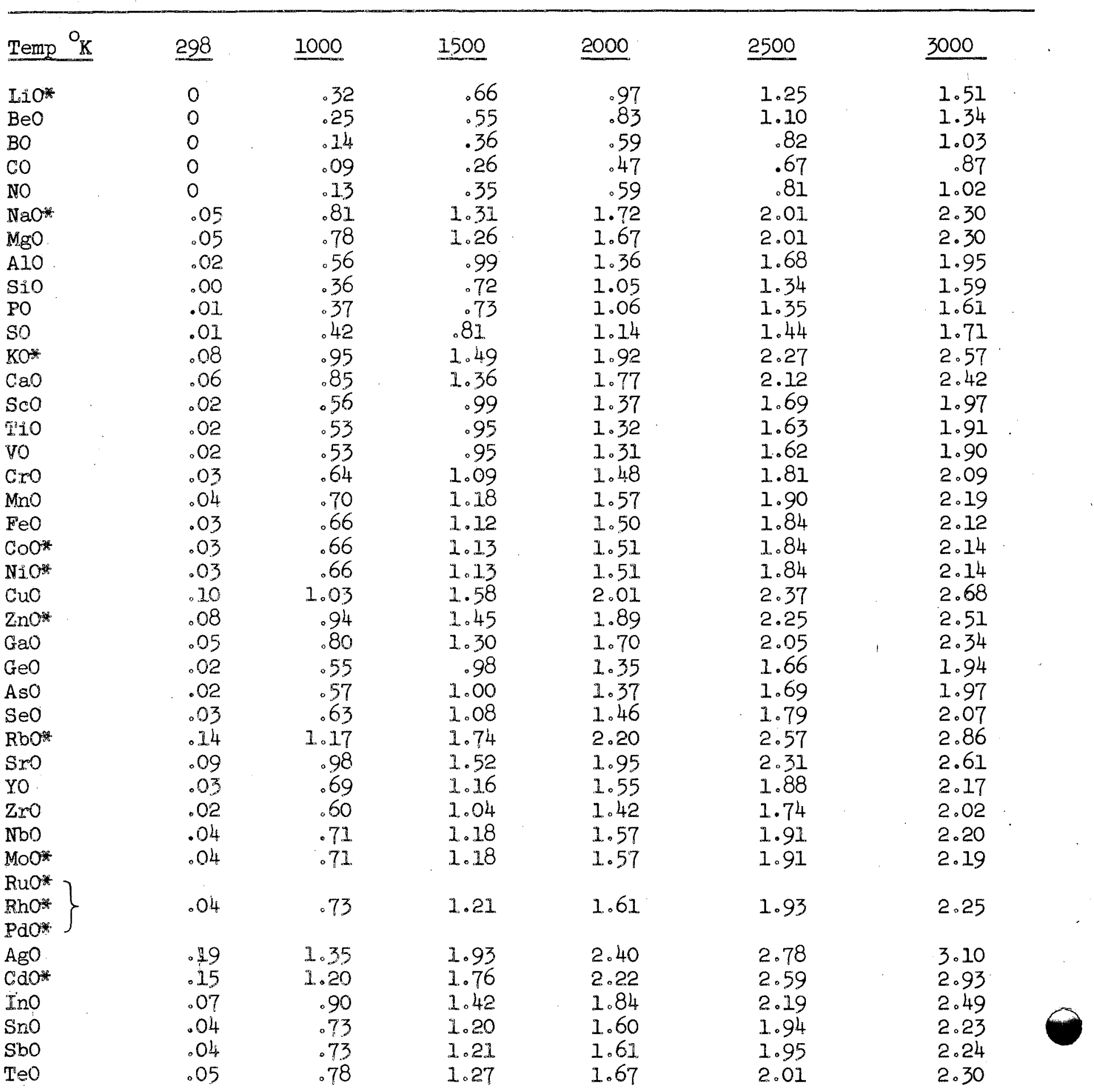


Table 4

(continued)

$$
-\left(\frac{F_{T}{ }^{\circ}-H_{0}^{O}}{T}\right)_{v i b} \text { for oxides }
$$

\begin{tabular}{|c|c|c|c|c|c|c|}
\hline Temp ${ }^{\circ} \mathrm{K}$ & 298 & 1000 & 1500 & 2000 & 2500 & 3000 \\
\hline $\mathrm{CsO}^{*}$ & .20 & 1.36 & 1.95 & 2.58 & 2.80 & 3.11 \\
\hline $\mathrm{BaO}$. & .08 & .95 & 1.48 & 1.91 & 2.27 & 2.57 \\
\hline $\mathrm{LaO}$ & .04 & .74 & 1.22 & 1.62 & 1.96 & 2.25 \\
\hline $\mathrm{HfO} *$ & .03 & .64 & 1.11 & 1.47 & 1.80 & 2.10 \\
\hline $\mathrm{TaO}^{*}$ & .03 & .64 & 1.09 & 1.47 & 1.80 & 2.08 \\
\hline WO* & .04 & .75 & 1.23 & 1.64 & 1.98 & 2.28 \\
\hline $\mathrm{ReO}^{*}$ & .05 & .81 & 1.31 & 1.72 & 2.06 & 2.37 \\
\hline $\mathrm{OsO}^{*}$ & & & & & & \\
\hline $\operatorname{IrO} O *\}$ & .05 & .78 & 1.26 & 1.68 & 2.01 & 2.34 \\
\hline $\mathrm{PtO}^{*} \mathrm{~J}$ & & & & & & \\
\hline $\mathrm{AuO} *$ & .3 & 1.58 & 2.20 & 2.67 & 3.08 & 3.38 \\
\hline $\mathrm{HgO} *$ & .21 & 1.38 & 1.98 & 2.46 & 2.84 & 3.14 \\
\hline T10* & .08 & 0.97 & 1.51 & 1.93 & 2.28 & 2.59 \\
\hline $\mathrm{PbO}$ & .06 & .87 & 1.38 & 1.80 & 2.15 & 2.44 \\
\hline $\mathrm{BiO}$ & .07 & .92 & 1.44 & 1.86 & 2.21 & 2.51 \\
\hline
\end{tabular}

*Estimated $\omega_{e}$ values by comparing with neighbors. 
Table 5

\begin{tabular}{|c|c|c|c|c|c|c|}
\hline & & $\left(\mathrm{F}_{\mathrm{T}}^{\mathrm{O}}\right.$ & $\int_{\operatorname{tr}}$ & on ${ }^{\text {for }}$ & & \\
\hline$\underline{T e m p}^{{ }^{K}}$ & $\underline{298}$ & 1000 & 1500 & 2000 & 2500 & 3000 \\
\hline LiO & 30.36 & 36.38 & 38.39 & 39.82 & 40.93 & 41.83 \\
\hline $\mathrm{BeO}$ & 30.62 & 36.63 & 38.65 & 40.08 & 41.19 & 42.09 \\
\hline BO & 30.83 & 36.84 & 38.86 & 40.29 & 41.39 & 42.30 \\
\hline $\mathrm{CO}$ & 30.96 & 36.97 & 38.99 & 40.41 & 41.52 & 42.43 \\
\hline No & 31.07 & 37.08 & 39.09 & 40.51 & 41.63 & 42.54 \\
\hline $\mathrm{NaO}$ & 31.95 & 37.96 & 39.97 & 41.40 & 42.51 & 43.42 \\
\hline $\mathrm{MgO}$ & 32.05 & 38.06 & 40.07 & 41.50 & 42.61 & 43.52 \\
\hline A.10 & 32.24 & 38.25 & 40.26 & 41.69 & 42.80 & 43.71 \\
\hline $\mathrm{SiO}$ & 32.31 & 38.32 & 40.34 & 41.77 & 42.88 & 43.78 \\
\hline PO & 32.50 & 38.51 & 40.53 & 41.96 & 43.07 & 43.97 \\
\hline SO & 32.57 & 38.58 & 40.60 & 42.03 & 43.13 & 44.04 \\
\hline $\mathrm{KO}$ & 32.97 & 38.99 & 41.00 & 42.43 & 43.53 & 44.44 \\
\hline $\mathrm{CaO}$ & 33.03 & 39.04 & 41.06 & 42.48 & 43.59 & 44.50 \\
\hline $\mathrm{ScO}$ & 33.28 & 39.30 & 41.31 & 42.73 & 43.84 & 44.75 \\
\hline Tio & 33.41 & 39.43 & 41.44 & 42.87 & 43.98 & 44.89 \\
\hline vo & 33.55 & 39.57 & 41.58 & 43.01 & 44.12 & 45.03 \\
\hline $\mathrm{CrO}$ & 33.60 & 39.62 & 41.63 & 43.06 & 44.17 & 45.07 \\
\hline $\mathrm{MnO}$ & 33.73 & 39.74 & 41.76 & 43.18 & 44.29 & 45.20 \\
\hline $\mathrm{FeO}$ & 33.76 & 39.78 & 41.79 & 43.22 & 44.33 & 45.24 \\
\hline $\mathrm{CoO}$ & 33.89 & 39.91 & 41.92 & 43.35 & 44.45 & 45.35 \\
\hline $\mathrm{NiO}$ & 33.88 & 39.90 & 41.91 & 43.34 & 44.45 & 45.35 \\
\hline $\mathrm{CuO}$ & 34.07 & 40.08 & $42 \cdot 10$ & 43.53 & 44.64 & 45.54 \\
\hline Zno & 34.14 & 40.15 & 42.17 & 43.59 & 44.70 & 45.61 \\
\hline $\mathrm{GaO}$ & 34.29 & 40.31 & 42.32 & 43.75 & 44.86 & 45.76 \\
\hline $\mathrm{GeO}$ & 34.39 & 40.40 & 42.42 & 43.85 & 44.96 & 45.86 \\
\hline $\mathrm{AsO}$ & 34.47 & 40.48 & 42.50 & 43.92 & 45.03 & 45.94 \\
\hline $\mathrm{SeO}$ & 34.60 & 40.61 & 42.63 & 44.05 & 45.16 & 46.07 \\
\hline $\mathrm{RbO}$ & 34.79 & 40.81 & 42.82 & 44.25 & 45.36 & 46.26 \\
\hline Sro & 34.86 & 40.87 & 42.89 & 44.31 & 45.42 & 46.33 \\
\hline YO & 34.89 & 40.91 & 42.92 & 44.35 & 45.46 & 46.36 \\
\hline Zro & 34.95 & 40.97 & $42 \cdot 98$ & 44.41 & 45.52 & 46.42 \\
\hline $\mathrm{NbO}$ & 35.00 & 41.02 & 43.03 & 44.46 & 45.57 & 46.47 \\
\hline MoO & 35.08 & 41.10 & 43.11 & 44.54 & 45.65 & 46.55 \\
\hline RuO & 35.23 & 41.25 & 43.26 & 44.69 & 45.79 & 46.70 \\
\hline RhO & 35.27 & 41.28 & 43.30 & 44.72 & 45.83 & 46.74 \\
\hline $\mathrm{PdO}$ & 35.36 & 41.38 & 43.39 & 44.82 & 45.93 & 46.83 \\
\hline $\mathrm{AgO}$ & 35.38 & 41.41 & 43.42 & 44.85 & 45.96 & 46.86 \\
\hline $\mathrm{CdO}$ & 35.49 & 41.51 & 43.52 & 44.95 & 46.06 & 46.96 \\
\hline Ino & 35.54 & 41.56 & 43.57 & 45.00 & 46.11 & 47.01 \\
\hline $\operatorname{Sn} O$ & 35.64 & 41.65 & 43.67 & $45 \cdot 10$ & 46.21 & 47.11 \\
\hline \multirow[t]{2}{*}{$\mathrm{SbO}$} & 35.71 & 41.72 & 43.74 & 45.16 & 46.27 & 47.18 \\
\hline & & & & & (conts & \\
\hline
\end{tabular}


Table 5

(continued)

$$
-\left(\frac{F_{T}^{0}-H_{0}^{0}}{T}\right)_{\text {translation }} \text { for oxides }
$$

\begin{tabular}{lllllll}
\hline Temp ${ }^{K}$ & 298 & 1000 & 1500 & 2000 & 2500 & 3000 \\
\hline TeO & 35.83 & 41.84 & 43.86 & 45.29 & 46.40 & 47.30 \\
CsO & 35.93 & 41.95 & 43.96 & 45.39 & 46.50 & 47.40 \\
BaO & 36.03 & 42.04 & 44.06 & 45.48 & 46.59 & 47.50 \\
LaO & 36.05 & 42.07 & 44.08 & 45.51 & 46.62 & 47.52 \\
CeO & 36.08 & 42.10 & 44.11 & 45.54 & 46.65 & 47.55 \\
SmO & 36.27 & 42.29 & 44.30 & 45.73 & 46.84 & 47.74 \\
EuO & 36.29 & 42.31 & 44.32 & 45.75 & 46.86 & 47.76 \\
GdO & 36.38 & 42.40 & 44.41 & 45.84 & 46.95 & 47.85 \\
LuO & 36.66 & 42.68 & 44.69 & 46.13 & 47.24 & 48.14 \\
HfO & 36.73 & 42.75 & 44.76 & 46.19 & 47.30 & 48.20 \\
TaO & 36.77 & 42.79 & 44.80 & 46.23 & 47.34 & 48.24 \\
WO & 36.81 & 42.83 & 44.84 & 46.27 & 47.38 & 48.28 \\
ReO & 36.85 & 42.87 & 44.88 & 46.31 & 47.42 & 48.32 \\
OsO & 36.90 & 42.92 & 44.93 & 46.36 & 47.47 & 48.37 \\
IrO & 36.95 & 42.97 & 44.98 & 46.41 & 47.52 & 48.42 \\
PtO & 36.98 & 42.99 & 45.01 & 46.44 & 47.55 & 48.45 \\
AuO & 37.00 & 43.02 & 45.03 & 46.46 & 47.57 & 48.47 \\
HgO & 37.05 & 43.07 & 45.08 & 46.51 & 47.62 & 48.52 \\
TIO & 37.11 & 43.12 & 45.14 & 46.56 & 47.67 & 48.58 \\
PbO & 37.15 & 43.16 & 45.17 & 46.60 & 47.71 & 48.62 \\
BiO & 37.17 & 43.18 & 45.20 & 46.63 & 47.74 & 48.64 \\
PoO & 37.18 & 43.20 & 45.21 & 46.64 & 47.75 & 48.65 \\
RaO & 37.38 & 43.40 & 45.41 & 46.84 & 47.95 & 48.86 \\
AcO & 37.39 & 43.41 & 45.42 & 46.85 & 47.96 & 48.87 \\
ThO & 37.46 & 43.48 & 45.49 & 46.92 & 48.03 & 48.94 \\
UO & 37.52 & 43.54 & 45.55 & 46.98 & 48.09 & 49.00
\end{tabular}


Table 6

Estimated - $\left(\frac{\mathrm{F}_{\mathrm{T}}^{{ }^{\mathrm{O}}-\mathrm{H}_{\mathrm{O}}{ }^{\circ}}}{\mathrm{T}}\right)$ in cal/deg mole for gaseous metallic diatomic oxides

\begin{tabular}{|c|c|c|c|c|c|c|}
\hline Temp ${ }^{\circ} \mathrm{K}$ & 298 & 1000 & 1500 & 2000 & $2500^{\circ}$ & 3000 \\
\hline LiO* & 43.2 & 51.9 & 55.1 & 57.4 & 59.2 & 60.7 \\
\hline $\mathrm{NaO}^{*}$ & 47.0 & 56.2 & 59.5 & 61.9 & 63.8 & 65.3 \\
\hline $\mathrm{MgO}$. & 43.8 & 53.0 & 56.3 & 58.7 & 60.6 & 62.1 \\
\hline $\mathrm{KO}^{*}$ & 49.1 & 58.4 & 61.8 & 64.2 & 66.1 & 67.7 \\
\hline $\mathrm{CaO}$ & 45.3 & 54.5 & 57.9 & 60.3 & 62.2 & 63.7 \\
\hline $\mathrm{ScO}$ & 48.8 & 58.4 & 61.6 & 64.1 & 66.0 & 67.7 \\
\hline TiO & 49.6 & 59.5 & 63.0 & 65.5 & 67.5 & 69.0 \\
\hline vo & 49.8 & 60.1 & 63.5 & 66.1 & 68.1 & 69.7 \\
\hline $\mathrm{CrO}$ & 49.4 & 60.1 & 63.6 & 66.0 & 68.1 & 69.8 \\
\hline $\mathrm{MnO}+$ & 49.4 & 58.5 & 61.8 & 64.2 & 66.2 & 67.6 \\
\hline $\mathrm{FeO}$ & 50.4 & 60.2 & 63.9 & 66.3 & 68.4 & 69.9 \\
\hline $\mathrm{COO}$ & 49.1 & 60.2 & 63.8 & 66.4 & 68.3 & 70.1 \\
\hline $\mathrm{NiO}$ & 50.3 & 59.5 & 63.1 & 65.6 & 67.7 & 69.4 \\
\hline $\mathrm{CuOt}$ & 50.1 & 59.5 & 62.9 & 65.5 & 66.6 & 69.1 \\
\hline $\mathrm{ZnO}$ & 46.5 & 55.8 & 59.1 & 61.5 & 63.4 & 65.0 \\
\hline GaOt & 47.8 & 57.0 & 60.3 & 62.7 & 64.6 & 66.1 \\
\hline $\mathrm{RbO*}$ & 51.6 & 61.1 & 64.4 & 67.0 & 68.8 & 70.4 \\
\hline $\mathrm{SrO}$ & $47 \cdot 7$ & 57.0 & 60.4 & 62.8 & 64.7 & 66.3 \\
\hline YO & 50.2 & 60.1 & 63.6 & 66.2 & 68.1 & 69.8 \\
\hline Zro & 50.6 & 60.6 & 62.7 & 67.0 & 69.0 & 70.6 \\
\hline $\mathrm{NbO}$ & 50.1 & 60.8 & 64.6 & 67.4 & 69.6 & 71.2 \\
\hline MoO* & 52.1 & 61.2 & 64.4 & 66.8 & 68.7 & 70.3 \\
\hline RuO* & 52.2 & 61.6 & 65.2 & 67.9 & 69.7 & 71.6 \\
\hline RhO* & 52.3 & 61.6 & 65.0 & 67.6 & 69.8 & 71.4 \\
\hline $\mathrm{PdO*}$ & 52.3 & 61.4 & 64.8 & 67.3 & 69.3 & 71.0 \\
\hline $\mathrm{AgOt}$ & 52.1 & 61.7 & 65.2 & 67.6 & 69.6 & 71.2 \\
\hline CdO* & 48.3 & 57.8 & 61.2 & 63.6 & 65.6 & 67.2 \\
\hline InOt & 49.6 & 58.9 & 62.2 & 64.6 & 66.5 & 68.1 \\
\hline $\mathrm{CsO}^{*}$ & 53.0 & 62.5 & 65.9 & 68.6 & 70.3 & 71.9 \\
\hline $\mathrm{LaO}+$ & 51.7 & 61.6 & 64.5 & 67.2 & 69.3 & 71.0 \\
\hline $\mathrm{HfO} *$ & 52.4 & 61.4 & 64.7 & 67.1 & 69.0 & 70.5 \\
\hline $\mathrm{TaO}$ & 53.6 & 62.7 & 65.9 & 68.3 & 70.2 & 71.7 \\
\hline WO* & 52.7 & 61.8 & 65.1 & 67.5 & 69.4 & 71.0 \\
\hline $\operatorname{ReO} *$ & .53 .2 & 62.4 & 65.7 & 68.1 & 70.0 & 71.6 \\
\hline $\mathrm{OsO}^{*}$ & 54.1 & 63.3 & 66.6 & 69.0 & 70.9 & 72.5 \\
\hline IrO* & 54.2 & 63.3 & 66.6 & 69.1 & 70.9 & 72.5 \\
\hline PtO* & 54.2 & 63.3 & 66.7 & 69.09 & 71.0 & 72.5 \\
\hline AuO* & 54.2 & 63.9 & 67.3 & 69.8 & 71.7 & 73.3 \\
\hline $\mathrm{HgO}^{*}$ & 50.3 & 69.9 & 63.3 & 65.8 & 67.7 & 69.3 \\
\hline TIO* & 51.5 & 60.8 & 64.2 & 66.61 & 68.5 & 70.1 \\
\hline Biot & 51.6 & 60.9 & 64.2 & 66.6 & 68.5 & 70.1 \\
\hline
\end{tabular}


Table 6a

Gaseous MO free energy functions known to better than $0.1 \mathrm{cal} / \mathrm{deg}$ mole $-\left(\frac{\mathrm{F}_{\mathrm{T}}{ }^{\mathrm{O}}-\mathrm{H}_{\mathrm{O}}{ }^{\circ}}{\mathrm{T}}\right)$ for $\mathrm{MO}(\mathrm{g})$

\begin{tabular}{lllllll}
\hline Temp ${ }_{\mathrm{K}}$ & \multicolumn{1}{l}{298} & $\underline{1000}$ & $\underline{1500}$ & $\underline{2000}$ & $\underline{2500}$ & $\underline{3000}$ \\
$\mathrm{~N}$ & & & & & & \\
$\mathrm{AlO}$ & 45.13 & 54.11 & 57.38 & 59.77 & 61.66 & 63.23 \\
$\mathrm{BO}$ & 41.66 & 50.21 & 53.26 & 55.51 & 57.30 & 58.79 \\
$\mathrm{BaO}$ & 49.02 & 58.31 & 61.66 & 64.08 & 66.00 & 67.57 \\
$\mathrm{BeO}$ & 40.24 & 48.92 & 52.06 & 54.37 & 56.20 & 57.72 \\
$\mathrm{CO}$ & 40.25 & 48.77 & 51.78 & 53.99 & 55.75 & 57.23 \\
$\mathrm{ClO}$ & 47.04 & 56.14 & 59.46 & 61.88 & 63.79 & 65.38 \\
$\mathrm{GeO}$ & 46.50 & 55.45 & 58.71 & 61.10 & 62.97 & 64.52 \\
$\mathrm{HO}$ & 36.82 & 45.39 & 48.30 & 50.41 & 52.11 & 53.52 \\
$\mathrm{NO}$ & 42.98 & 51.86 & 54.96 & 57.24 & 59.04 & 60.55 \\
$\mathrm{OO}$ & 42.06 & 50.70 & 53.81 & 56.10 & 57.94 & 59.48 \\
$\mathrm{PbO}$ & 50.16 & 59.38 & 62.71 & 65.13 & 67.03 & 68.59 \\
$\mathrm{SO}$ & 46.03 & 54.87 & 58.09 & 60.43 & 62.30 & 63.85 \\
$\mathrm{SiO}$ & 43.55 & 52.32 & 55.51 & 57.84 & 59.70 & 61.25 \\
$\mathrm{SnO}$ & 48.35 & 57.47 & 60.77 & 63.19 & 65.09 & 66.65 \\
& & & & & & \\
\hline
\end{tabular}

The values for $\mathrm{AlO}, \mathrm{BO}, \mathrm{BeO}, \mathrm{CO}, \mathrm{ClO}, \mathrm{HO}, \mathrm{NO}, \mathrm{O}_{2}$, and $\mathrm{sO}$ are taken from Appendix 7 of Pitzer and Brewer, "Thermodynamics," McGraw-Hill, N. Y. (196I).

Most of the values in Table Gare slightly larger at high temperatures than the values obtained from Tables $1-5$ due to inclusion of anharmonic and stretching terms. 
This report was prepared as an account of Government sponsored work. Neither the United States, nor the Commission, nor any person acting on behalf of the Commission:

A. Makes any warranty or representation, expressed or implied, with respect to the accuracy, completeness, or usefulness of the information contained in this report, or that the use of any information, apparatus, method, or process disclosed in this report may not infringe privately owned rights; or

B. Assumes any liabilities with respect to the use of, or for damages resulting from the use of any information, apparatus, method, or process disclosed in this report.

As used in the above, "person acting on behalf of the Commission" includes any employee or contractor of the Commission, or employee of such contractor, to the extent that such employee or contractor of the Commission, or employee of such contractor prepares, disseminates, or provides access to, any information pursuant to his employment or contract with the Commission, or his employment with such contractor. 